\title{
Vessel Surface Reconstruction With a Tubular Deformable Model
}

\author{
Peter J. Yim*, Juan J. Cebral, Rakesh Mullick, Hani B. Marcos, and Peter L. Choyke
}

\begin{abstract}
Three-dimensional (3-D) angiographic methods are gaining acceptance for evaluation of atherosclerotic disease. However, measurement of vessel stenosis from 3-D angiographic methods can be problematic due to limited image resolution and contrast. We present a method for reconstructing vessel surfaces from 3-D angiographic methods that allows for objective measurement of vessel stenosis. The method is a deformable model that employs a tubular coordinate system. Vertex merging is incorporated into the coordinate system to maintain even vertex spacing and to avoid problems of self-intersection of the surface. The deformable model was evaluated on clinical magnetic resonance $(\mathrm{MR})$ images of the carotid $(n=6)$ and renal $(n=2)$ arteries, on an MR image of a physical vascular phantom and on a digital vascular phantom. Only one gross error occurred for all clinical images. All reconstructed surfaces had a realistic, smooth appearance. For all segments of the physical vascular phantom, vessel radii from the surface reconstruction had an error of less than 0.2 of the average voxel dimension. Variability of manual initialization of the deformable model had negligible effect on the measurement of the degree of stenosis of the digital vascular phantom.
\end{abstract}

Index Terms-Atherosclerosis, deformable model, magnetic resonance angiography, surface reconstruction.

\section{INTRODUCTION}

A THEROSCLEROSIS is assessed according to the degree of stenosis of diseased vessels. For example, stenosis of the carotid artery of greater than $70 \%$ represents a high risk of stroke and should be corrected by endarterectomy [1]. The standard method for measuring the degree of stenosis is digital subtraction angiography (DSA) which provides high-contrast projection images of vessels from multiple viewpoints. However, DSA requires intra-arterial placement of a catheter that can have serious medical complications. Thus, alternatives to DSA have been proposed including magnetic resonance angiography (MRA) [2], three-dimensional (3-D) ultrasound [3], and computed tomographic angiography (CTA) [4]. These methods are attractive because they are all less invasive than DSA and provide 3 -D images of the vessel lumen. However, the images from

Manuscript received October 23, 2000; revised September 26, 2001. The Associate Editor responsible for coordinating the review of this paper and recommending its publication was W. Higgins. Asterisk indicates corresponding author.

*P. J. Yim is with the Imaging Sciences Program, National Institutes of Health, Bldg. 10, Room 1C660, Bethesda, MD 20892 USA (e-mail: pyim@nih.gov).

J. J. Cebral is with the School of Computational Sciences, George Mason University, Fairfax, VA 22030 USA.

R. Mullick, GE John F. Welch Technology Center, Bangalore, India.

H. B. Marcos and P. L. Choyke are with the Imaging Sciences Program, National Institutes of Health, Bethesda, MD 20892 USA.

Publisher Item Identifier S 0278-0062(01)11146-8. these methods are significantly more difficult to interpret due to lower image resolution and contrast.

In this paper, we will present a computational method for reconstruction of vessel shape from 3-D angiography. This method allows for an objective evaluation of vessel shape and may improve the precision of shape measurements from 3-D angiography. Furthermore, reconstruction of the complete vascular surface provided allows for more sophisticated characterization of vessel shape at stenoses. Finally, surface reconstruction is useful for accurate computational fluid dynamics (CFD) modeling of arterial blood flow [5]-[7]. Such modeling provides estimates of fluid-mechanical conditions in the vessel that may be a factor in the progression of atherosclerosis [8], [9].

\section{BACKGROUND}

A variety of methods have been applied to the problem of vessel-surface reconstruction from 3-D angiography. We will review these methods in this section and discuss their relation to the problem of evaluation of atherosclerosis.

The simplest method for reconstruction of vessel surfaces is iso-surface reconstruction. In this method, the location of the surface is determined strictly according to image intensity. A smooth surface is produced by placing the vertices at fractional image grid locations such that the interpolated image intensity is constant on the surface [10]. However, choice of the threshold or iso-intensity level may be problematic, particularly for MRA, due to image inhomogeneities and other artifacts [11]. Thus, the use of the iso-intensity surface is restricted to qualitative applications.

Surface reconstruction of vessels has also been obtained from segmentation methods. Segmentation methods incorporate spatial and intensity criteria to objectively classify regions or voxels in an image. For example, a $k$-means clustering method automatically identifies voxels within vessels from MRA. This method compensates for the partial volume effect that diminishes the intensity of the smaller vessels thus causing errors for methods based on image intensity alone [12]. Another method uses "fuzzy connectivity" for separating arteries and veins in MRA obtained with blood pool contrast agents [13]. The arteries and veins are separated from the arteries based on a limited number of seed points inside and outside of the vessels. A similar watershed method has been successfully applied to MRA of the thoracic aorta [14], [15].

Once the image has been segmented surfaces can be readily composed from segmentation boundaries although considerable 
smoothing must be applied [15], [5], [16], [17] and topological errors must be corrected [16]. However, these segmentation methods incorporate only very limited a priori knowledge of the anatomy. Thus, while the shapes created by these methods directly reflect the information in the images they tend to suffer unnecessarily from errors due to image artifacts or image noise. Several model-based approaches have been taken to address this problem.

Several multiscale methods have been applied to the problem of vessel surface reconstruction [18]-[20]. In these methods, the width of objects in grey-scale images are automatically determined for any given point in the image by matching the objects with convolution kernels of various sizes. Thus, for points along a vessel, an average vessel diameter is obtained. Together with the vessel axis, the diameter measurements fully describe the vessel surface. However, all such multiscale methods for vessel surface reconstruction assume that the vessel has a symmetric cross-sectional shape. Therefore, these methods have limited application for reconstruction of the vessel shape near bifurcations or in the presence of vascular disease such as atherorsclerosis.

Another class of methods, the deformable models, allows for more general vessel shapes while still providing the useful smoothing effects. In the deformable models, surfaces deform according to image and smoothing "forces" [21]. The image forces pull the surface toward edges in the image while the smoothing forces resist bending of the surface and maintain even spacing of vertices of the surface mesh. Several deformable models have been applied to reconstruction of vessel surfaces. Two-dimensional methods have been proposed in which the vessel boundary is reconstructed independently for each slice or plane [6], [7], [22]. These provide adequate results for the cases tested but improvements can certainly be obtained by unified 3-D reconstruction methods.

Several general-purpose 3-D deformable models have shown promise for surface reconstruction of large vessels. These methods have the potential to reconstruct nontubular shapes such as vessel aneurysms and to provide an integrated reconstruction of bifurcations from minimal initializations [23]-[25]. However, gross errors from these surface reconstructions have been found to occur in the region of bifurcations.

Recently, a 3-D deformable model has been developed by Frangi et al. specifically for vessel surface reconstruction that is based on a cylindrically parametrized surface mesh [26]. Since the cylindrical mesh is well-suited for vessel shape, a greater degree of smoothing can be obtained than from more generalized deformable model meshes. The model is initialized by a multiscale method in which the axis is constructed while simultaneously estimating vessel diameter. Once the deformable model is initialized, the mesh deforms according to the conventional mechanical analogy. However, there are inherent limitations in this deformable model. One limitation is that the smoothing constraints used in this model inherently bias the surface reconstruction. For example, the "stretching" force on the surface vertices, which is meant to produce an even spacing of vertices, also tends to constrict the vessel. While a "bending" force is imposed to counteract that constriction effect, a complete cancellation cannot be obtained. Another inherent problem of this deformable model is the limited maximum density of vertices

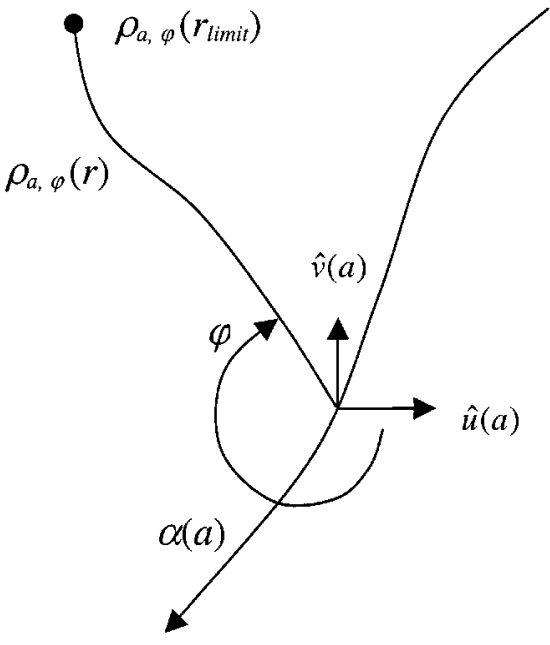

Fig. 1. Definition of parameters of tubular coordinate system. Unlike a cylindrical coordinate system, the axial and radial lines of the tubular coordinate system may be curvilinear. The axis and the radial lines of the tubular deformable model are paths $\alpha$ and $\rho$ parametrized by the variables $a$ and $r$, respectively. $\hat{u}(a)$ and is the reference radial direction at each axial location. $\hat{v}(a)$ is orthogonal to $\hat{u}(a) . \varphi$ is the angular location of each radial line.

in the surface mesh. While this model can be readily initialized for a low density of vertices, surface self-intersection problems will occur if the density of vertices is increased in the axial direction. We will discuss this problem in greater detail in the next section. We will present a new deformable model that resolves these problems.

\section{The Tubular Deformable Model}

\section{A. The Deformation Process}

The model we present allows for curves in the vessel axis, variability in the vessel diameter and variability in cross-sectional shape. In our deformable model, vessel shape is described by a radial function of axial and circumferential position, $R_{i}(a, \varphi), N^{2} \rightarrow \mathbf{R}$. The parameter $a$ designates the axial location and the parameter $\varphi$ designates the circumferential location. The subscript $i$ indicates the iteration of deformable model computation.

Parameters of the radial function $R_{i}(a, \varphi)$, are defined within a tubular coordinate system which resembles but is not identical to a cylindrical coordinate system. In particular, the tubular coordinate system accommodates curved axes. However the tubular coordinate system reduces to the cylindrical coordinate system for straight axes. The axis of the tubular coordinate system is described by a path $\alpha: D_{\alpha} \subset N \rightarrow R^{3}$. The radial lines at each axial and circumferential position are described by the paths $\rho_{a \varphi}: D_{\rho} \subset N \rightarrow R^{3}$. The construction of the axial and radial paths will be described in more detail in the following sections. The notation of the coordinate system is clarified in Fig. 1.

The deformable model is represented by a surface mesh. The vertices of the mesh fall at evenly spaced locations in the axial and circumferential directions.

The radial function $R_{i}(a, \varphi)$ describes the location of each vertex relative to the tubular coordinate system. The initial value 


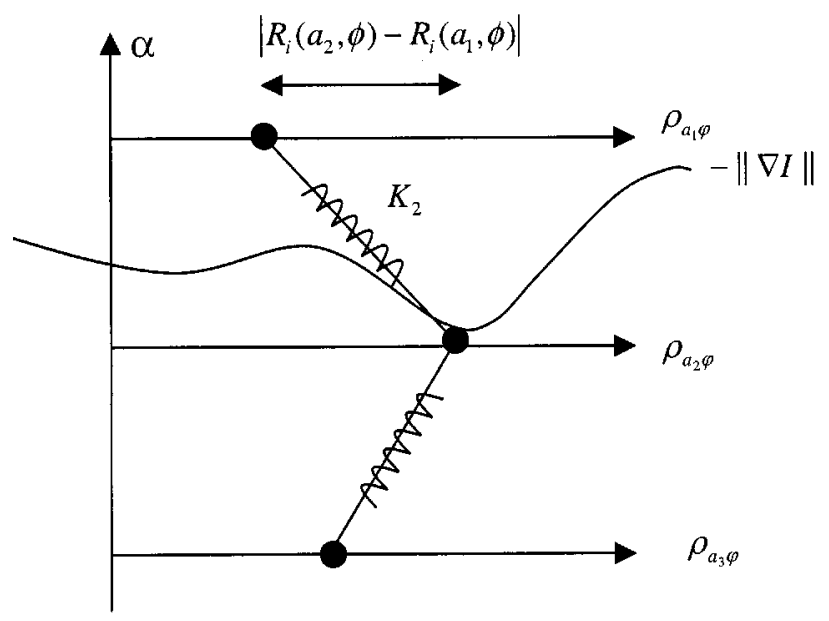

Fig. 2. Deformation of vertices of surface mesh. All vertices of the surface deform along radial lines $\rho_{a_{1} \varphi}, \rho_{a_{2} \varphi}, \rho_{a_{3} \varphi}$. The negative gradient magnitude image provides a gravitational force on the vertex (curved line) while the neighboring vertices pull the vertex with spring forces. The spring forces are proportional to the difference in the radial locations between the vertices. (The implied $y$-axis of the gravitational force curve is $-\|\nabla I\|$.)

of the radial function $R_{0}(a, \varphi)$ is the value of the radial parameter $r$ at the maxima of the gradient magnitude.

$$
R_{0}(a, \varphi)=\arg \max _{r}\left\|\nabla I\left(\rho_{a, \phi}(r)\right)\right\| .
$$

A further provision is that a decrease in image intensity occurs at the point, $R_{0}(a, \varphi)$ proceeding outwards from the vessel center

$$
\nabla I\left(\rho_{a \varphi}\left(R_{0}(a, \phi)\right)\right) \cdot \rho_{a \varphi}^{\prime}\left(R_{0}(a, \phi)\right)>0 .
$$

$\nabla I$ is obtained by convolution of the image with the gradient of the normalized spherical Gaussian function. The scale of the Gaussian kernel is dependent on the application.

After the radial function, $R(a, \varphi)$, is initialized it deforms according to image and smoothing forces. The deformation is analogous to a mechanical equilibration process. As such, the instantaneous deformation of each vertex is given by

$$
\begin{aligned}
R_{i+1}(a, \phi)=R_{i}(a, \phi)+K_{1}( & K_{2} \sum_{a_{n}^{\prime} \phi_{n}}\left(R_{i}\left(a_{n}, \phi_{n}\right)\right. \\
& \left.-R_{i}(a, \phi)\right)+\frac{\rho_{a \varphi}^{\prime}\left(R_{i}(a, \phi)\right)}{\left\|\rho_{a \varphi}^{\prime}\left(R_{i}(a, \phi)\right)\right\|} \\
& \left.\cdot \nabla\left\|\nabla I\left(\rho_{a \varphi}\left(R_{i}(a, \varphi)\right)\right)\right\|\right)
\end{aligned}
$$

where $a_{n}$ and $\varphi_{n}$ are the axial and angular locations adjacent to $a$ and $\phi . K_{1}$ and $K_{2}$ are constants that are determined by trial-and-error for a given class of images. $K_{1}$ represents the temporal step size of the deformation process (in units of distance). $K_{2}$ represents the weight or importance given to the smoothing force relative to the image force (in units of inverse distance). The deformations are applied simultaneously to all vertices and repeated until an equilibrium condition is obtained. The mechanical analogy of the equilibration process is illustrated in Fig. 2.
Additional forces may be included in the deformation process. For example, forces may be applied through user interaction to correct for gross errors in the surface reconstruction. An "anchor" manually placed at location $\left(a_{\text {manual }}, \varphi_{\text {manual }}, r_{\text {manual }}\right)$ will attract the surface toward itself. The manual force is implemented by modifying (3) to include a manual-force term

$$
\begin{array}{r}
R_{i+1}(a, \phi) \\
=R_{i}(a, \phi)+K_{1}\left(K_{2} \sum_{a_{n}^{\prime} \phi_{n}}\left(R_{i}\left(a_{n}, \phi_{n}\right)-R_{i}(a, \phi)\right)\right. \\
+\frac{\rho_{a \varphi}^{\prime}\left(R_{i}(a, \phi)\right)}{\left\|\rho_{a \varphi}^{\prime}\left(R_{i}(a, \phi)\right)\right\|} \\
\left.\cdot \nabla\left\|\nabla I\left(\rho_{a \varphi}\left(R_{i}(a, \varphi)\right)\right)\right\|\right) \\
+\delta\left(a_{\text {manual }}, \varphi_{\text {manual }}\right) K_{1} K_{3}\left(R_{i}(a, \phi)-r_{\text {manual }}\right)
\end{array}
$$

here

$$
\begin{aligned}
& \delta\left(a_{\text {manual }}, \varphi_{\text {manual }}\right) \\
& =1, \quad \text { for }(a, \varphi)=\left(a_{\text {manual }}, \varphi_{\text {manual }}\right) \\
& =0, \quad \text { for }(a, \varphi) \neq\left(a_{\text {manual }}, \varphi_{\text {manual }}\right)
\end{aligned}
$$

\section{B. The Tubular Coordinate System}

The deformable model of the vessels is based on a tubular coordinate system that allows for curvature of the cylindrical axis. The tubular coordinate system differs from the cylindrical coordinate system in several respects. First, the circumferential coordinate of the tubular coordinate system does not correspond to an absolute direction. Rather, the absolute direction for a given circumferential coordinate varies along the axis depending on curvature of the axis. We define a circumferential coordinate such that a minimum of angular change occurs between adjacent points on the axis. Given the path of the vessel, $\alpha(a), \hat{w}(a)$ is the unit vector along the vessel axis and $\hat{u}(a)$ is the reference radial orientation. These vectors are shown in Fig. 1

$$
\begin{aligned}
\hat{w}(a) & \equiv \frac{\alpha^{\prime}(a)}{\left\|\alpha^{\prime}(a)\right\|} \\
\hat{u}(a) & \equiv \frac{\rho_{a 0}^{\prime}(0)}{\left\|\rho_{a 0}^{\prime}(0)\right\|} .
\end{aligned}
$$

The reference orientation at the adjacent axial point is then

$$
\hat{u}(a+1)=\frac{\hat{u}(a)-\hat{w}(a+1)((\hat{u}(a) \cdot \hat{w}(a+1))}{\| \hat{u}(a)-\hat{w}(a+1)((\hat{u}(a) \cdot \hat{w}(a+1)) \|} .
$$

The reference circumferential orientation for a curved axis is shown in Fig. 3. A normal radial vector is obtained by the cross product of the reference radial vector with the axis vector

$$
\hat{v}(a) \equiv \hat{u}(a) \times \hat{w}(a) .
$$

All initial radial vectors are constructed from a linear combination of the reference and normal radial directions. Our imple- 


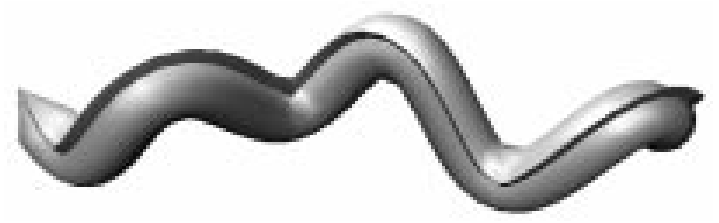

Fig. 3. Reference orientation for circumferential coordinate angle. The reference angle on which the circumferential angle is based is dependent on the curvature of the vessel axis.

mentation of the tubular deformable model uses 16 radial lines at each axial location evenly spaced circumferentially.

Thus, the radial vector $\hat{v}$ corresponds to a unit vector at the 4 th angular position and 0 th radial position $(\varphi=4$ and $r=0)$

$$
\hat{v}(a)=\frac{\rho_{a 4}^{\prime}(0)}{\left\|\rho_{a 4}^{\prime}(0)\right\|}
$$

A second feature of the tubular coordinate system, as mentioned in the previous section, is that the radii do not emanate in straight lines from the axis at all points. Rather, the radial lines are warped in areas where the vessel axis is curved. This warping prevents radial lines from adjacent axial locations from intersecting one another.

The radial lines are defined prior to the deformation process. The radial lines extend outwards at a constant step size (Step_size) of 0.1 of the in-plane pixel resolution. Spatial resolution below this step size is considered insignificant

$$
\rho_{a \varphi}^{\text {initial }}(r)=\alpha(a)+\frac{\rho_{a \phi}^{\prime}(0)}{\left\|\rho_{a \phi}^{\prime}(0)\right\|}(r)(\text { Step_size }) .
$$

Radial lines are concatenated when intersections would occur. In principle, the radial lines from an axis will not intersect one another exactly due to the discrete locations of the radial lines and to out-of-plane components of the radial direction vectors. Thus, a practical definition of line intersection must be adopted. Radial lines are considered to intersect one another if one radial line enters the territory of the second radial line. The territory, $T_{a \varphi}$ of a radial line is the region that is closer to the origin of that radial line than to that of any other radial line

$$
T_{a \varphi} \equiv\left\{p \in R^{3} \mid\|p-\alpha(a)\|<\|p-\alpha(b)\|, \forall b \in D_{\alpha} \backslash a\right\} .
$$

Thus, for a given circumferential location, territories of all radial lines are nonoverlapping or nonintersecting

$$
T_{a \varphi} \cap T_{b \varphi}=\emptyset, \quad \forall(a, b) \in D_{\alpha} \times D_{\alpha}, \quad a \neq b .
$$

The concept of territories can be used to prevent intersections of radial lines by confining each radial line to its own territory. This principle is illustrated in Fig. 4.

In practice, the definition of territories by (11) is overly conservative. If each radial line is strictly confined to its own territory, gaps occur between radial lines. Gaps can be eliminated by relaxing the definition of territories to allow for a modest degree of overlap of the territories

$$
T_{a \varphi}^{\text {modified }} \equiv T_{a \varphi} \cup T_{a \varphi}^{-} \cup T_{a \varphi}^{+}
$$

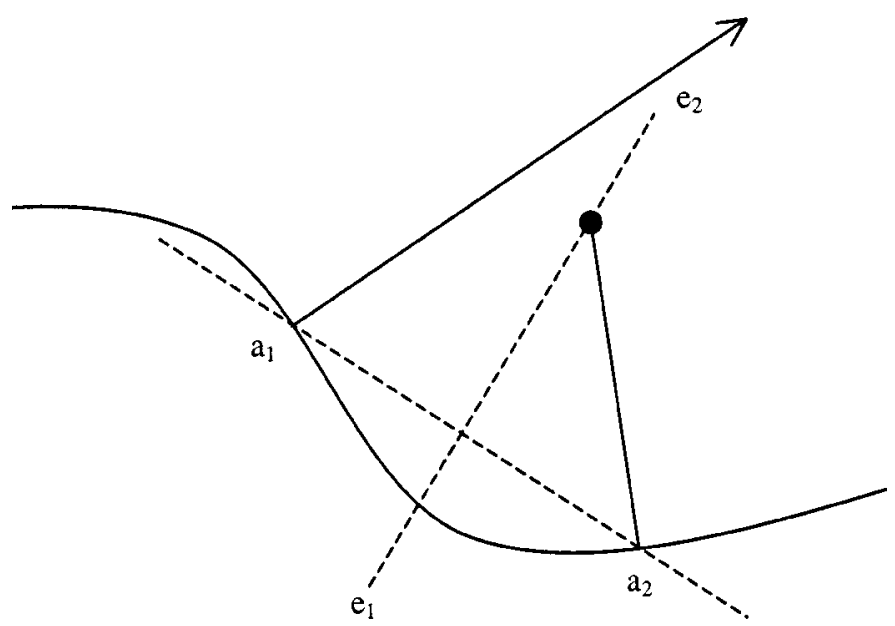

Fig. 4. Condition for truncation of radial lines. Radial lines project outwards perpendicularly from the predefined axis of the tubular coordinate system. In certain cases the radial lines must be truncated to prevent intersections. The condition for truncation is that a radial line leaves its "territory". In this diagram, the radial line from the axial position $a_{2}$ is truncated when it enters the territory of $a_{1}$. The boundary between two territories is located at the line of equidistance between $a_{1}$ and $a_{2}\left(e_{1} e_{2}\right)$. Truncation of radial lines from nonadjacent axial positions (11) is shown.

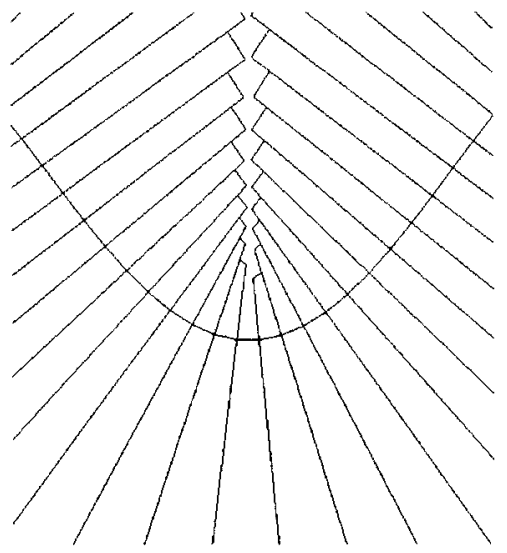

Fig. 5. The tubular coordinate system. Radial lines from an axis will intersect at the inside of axis curves. This problem is solved by merging radial lines in the tubular coordinate system. Radial lines are shown for the in-plane radial directions along a curved portion of the axis of a tubular coordinate system. The radial coordinate at each point in the tubular coordinate system is the distance to the nearest point on the axis.

where $T_{a \varphi}^{-}$and $T_{a \varphi}^{+}$are portions of the territories of the adjacent radial lines

$$
\begin{aligned}
& T_{a \varphi}^{-} \equiv\left\{t \in T_{(a-1) \varphi}\|t-\alpha(a, \varphi)\|<\left\|x_{a \varphi}^{-}-\alpha(a, \varphi)\right\|\right\} \\
& \text { for } a>1 \\
& =\emptyset \text { for } a=1 \\
& T_{a \varphi}^{+} \equiv\left\{t \in T_{(a+1) \varphi}\|t-\alpha(a, \varphi)\|<\left\|x_{a \varphi}^{+}-\alpha(a, \varphi)\right\|\right\} \\
& \text { for } a<a_{\max } \\
& =\emptyset \text { for } a=a_{\max } \text {. }
\end{aligned}
$$

The points $x_{a \varphi}^{-}$and $x_{a \varphi}^{+}$are defined as points on a given radial line $\rho_{a \varphi}$ at the point at which a radial line most nearly intersects with the radial lines $\rho_{(a-1) \varphi}$ and $\rho_{(a+1) \varphi}$, from downstream and 


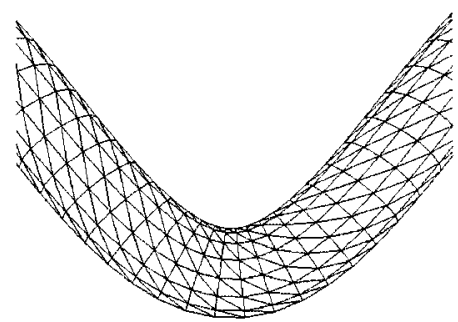

(a)

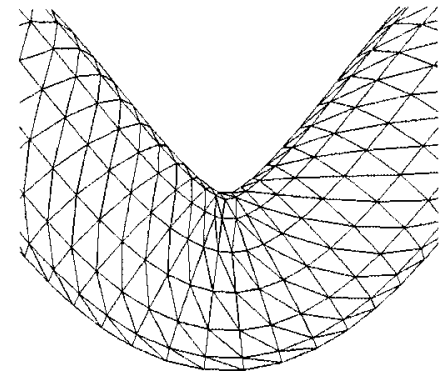

(b)

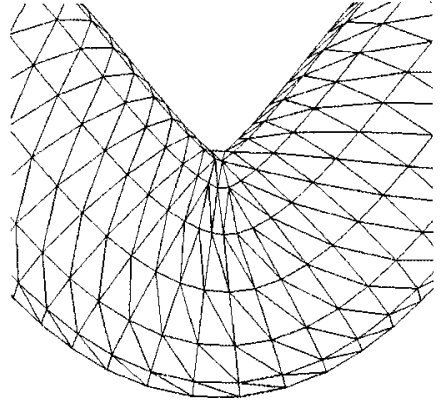

(c)

Fig. 6. Iso-distance surfaces. Surfaces are constructed whose vertices are at equal distances from the given axis. The meshing is done according to the tubular coordinate system. For small radii (a), vertices exist for each circumferential and axial position. At larger radii, the vertices merge with one another in the axial direction to eliminate criss-crossing of radial lines (b) and (c).

upstream points along the axis, respectively. When these intersection points exist, the following is true:

$$
x_{a \varphi}^{-} \approx x_{(a-1) \varphi}^{+} \text {for } 1<a \leq a_{\max } .
$$

These intersections occur only if the radial lines $\rho_{a \varphi}$ and $\rho_{(a-1) \varphi}$ are convergent. If radial lines $\rho_{a \varphi}$ and $\rho_{(a-1) \varphi}$ are not convergent then

$$
x_{a \varphi}^{-} \equiv \alpha(a, \varphi) \quad \text { and } \quad x_{(a-1) \varphi}^{+} \equiv \alpha(a-1, \varphi) .
$$

The truncation point for each radial line is then defined as the point at which the radial line leaves its own territory

$$
\rho(r) \in T_{a \varphi}^{\text {modified }}, \quad \forall r<r_{a \varphi}^{\text {trunc }} .
$$

By the modified definition of territories, truncations tend to occur only between radial lines from nonadjacent points on the axis.

After the process of truncation, truncated radial lines then merged so that all extend outwards to the maximal radial position. The merging proceeds according to the following algorithm:

$$
\begin{aligned}
& \text { For } r=r_{\text {limit }}-1 \text { to } r=1 \\
& \text { For all }(a, \varphi) \\
& \text { if } r_{a \varphi}^{\text {trunc }}=r \\
& r_{a \varphi}^{\text {trunc }}=r_{\text {limit }} \\
& \text { for } \tilde{r}=r \text { to } r_{\text {limit }} \\
& \rho_{a \varphi}(\tilde{r})=\rho_{a_{\text {ncarcst }} \varphi}(\tilde{r}) \\
& \rho_{a \varphi}^{\prime}(\tilde{r})=\rho_{a_{\text {ncarest } \varphi}}^{\prime}(\tilde{r})
\end{aligned}
$$

$r_{\text {limit }}$ is the predefined maximal value of the parameter of the radial paths. $a_{\text {nearest }}$ is the axial location of the nonconcatenated radial line that is nearest in the axial direction such that

$$
r_{a_{\text {ncarcst }} \varphi}^{\text {trunc }}=r_{\text {limit }} .
$$

A set of radial paths for a segment of an axis are shown in Fig. 5. Meshes constructed from vertices at constant radial locations for a curved axis are shown in Fig. 6.

\section{Merging Vertices}

The cylindrical deformation process could be applied directly to the modified cylindrical coordinate system. However, bunching of vertices will occur where radial lines merge. Where such bunching occurs, the effective elasticity of the surface becomes significantly greater which reduces the surface smoothness. This effect is removed by merging vertices in the deformation process to match the merging of the radial lines. This precludes two vertices from co-existing on the same radial line.

Before describing the procedure for merging vertices, we introduce a notation for describing vertices. The vertices form a set $X=\left\{X_{1,1}, X_{1,2}, \ldots X_{2,1}, X_{2,2}, \ldots X_{a, \varphi} \ldots X_{K, 16}\right\}$ where first vertex subscript refers to the axial location and the second subscript refers to the angular location. There is a single radial path associated with each vertex. Also associated with each vertex is the number of vertices with which it is merged: $M: X \rightarrow N$. Initially, $M\left(X_{a, \varphi}\right)=1$ for all vertices.

The procedure for merging vertices is as follows. The vertices initially exist for all circumferential and axial locations. After a primary deformation phase, vertices are merged with their axial neighbors if either of the vertices is at a point where the radial lines of the two vertices are merged.

Merge two vertices $X_{a_{1}, \varphi}$ and $X_{a_{2}, \varphi}$ (at locations $\left(a_{1}, \varphi\right)$ and $\left(a_{2}, \varphi\right)$, respectively)

$$
\text { if } \rho_{a_{1} \varphi}\left(r_{1}\right)=\rho_{a_{2} \varphi}\left(r_{1}\right) \quad \text { or if } \quad \rho_{a_{1} \varphi}\left(r_{2}\right)=\rho_{a_{2} \varphi}\left(r_{2}\right)
$$

where

$$
r_{1}=R_{i}\left(a_{1}, \varphi\right) \quad \text { and } \quad r_{2}=R_{i}\left(a_{2}, \varphi\right) .
$$

There are several effects of merging of two vertices. Firstly, the vertices henceforth move in tandem. The radial location of the merged vertices is the average of each component of the merged vertices

$$
R_{i+1}\left(a_{1}, \varphi\right)=R_{i+1}\left(a_{2}, \varphi\right)=\frac{\sum_{m \in M_{a_{1}, \varphi}^{i+1}} R_{i}\left(a_{m}, \varphi\right)}{n\left(M_{a_{1}, \varphi}^{i+1}\right)} .
$$

$X_{a_{1}, \varphi}$ and $X_{a_{2}, \varphi}$ now share the same set of merged vertices.

$$
M_{a_{1}, \varphi}^{i+1}=M_{a_{2}, \varphi}^{i+1}=M_{a_{1}, \varphi}^{i} \cup M_{a_{2}, \varphi}^{i} .
$$

The set of circumferential neighbors accumulates for each merging such that the number of circumferential neighbors outnumbers the axial neighbors. To maintain a balance between the axial and circumferential component of the elastic force, the 
total circumferential elastic force must be rescaled. Thus, (3) is modified as follows for the merged phase of deformations:

$$
\begin{aligned}
& R_{i+1}(a, \phi) \\
& =R_{i}(a, \phi)+K_{1} \\
& \quad \times\left(\sum _ { a _ { n } \phi _ { n } } \left(K_{2_{m}}^{a, \varphi}\left(R_{i}\left(a_{n}, \phi_{n}\right)-R_{i}(a, \phi)\right)\right.\right. \\
& \left.\quad+\frac{\rho_{a \varphi}^{\prime}\left(R_{i}(a, \phi)\right)}{\left\|\rho_{a \varphi}^{\prime}\left(R_{i}(a, \phi)\right)\right\|} \cdot \nabla\left\|\nabla I\left(\rho_{a \varphi}\left(R_{i}(a, \varphi)\right)\right)\right\|\right) .
\end{aligned}
$$

The deformations in the merged phase is exactly the same as in the initial phase except that elasticity $K_{2}$ is different in the circumferential and axial directions for merged vertices. For axial neighbors, the elasticity constant has the same value as for the initial deformation phase

$$
K_{2 m}^{a, \varphi}=K_{2}
$$

For the circumferential neighbors, the elasticity constant is reduced according to the following formula:

$$
K_{2_{m}}^{a, \varphi}=\frac{K_{2}}{n\left(M_{a, \varphi}^{i}\right)}
$$

\section{Formation of Vessel Axis}

The tubular coordinate system is constructed from a vessel axis that is determined manually. This step could potentially be carried out in an automated or semi-automated manner [18], [26], [32]. However, robustness of existing methodologies is not established for the carotid artery. Factors that complicate centerline detection in the carotid artery bifurcation region include image artifacts such as venous contamination in contrast-enhanced MRA and proximity of the internal and external carotid arteries distal to the bifurcation. Also, centerlines are subject to error in bifurcation regions where the vessel path is inherently ambiguous [18], [26]. Further discussion of centerline detection or skeletonization is beyond the scope of this investigation.

The axis is formed from a sequence of points along the center of the vessel identified by the user. A sufficient number of points must be chosen to represent all the curves in the vessel. Points along the center of the vessel can be easily identified by the user by displaying slices of the image normal to the axis of the vessel side-by-side with a 3-D surface display of the vessels to provide anatomical reference (Fig. 7). In certain cases where the vessel follows a torturous path, points along the axis may be chosen from the maximum intensity projection (MIP). Identification of points on a MIP provides a 3-D coordinate since each point in the MIP is associated with a single point in the 3-D image in the projection process. The axis is smoothed with a cubic b-spline and interpolated to have a spacing of $0.5 \mathrm{~mm}$ between points. Thus, the spacing of the vertices in the circumferential and axial directions is similar for vessels of the dimensions of the carotid artery.

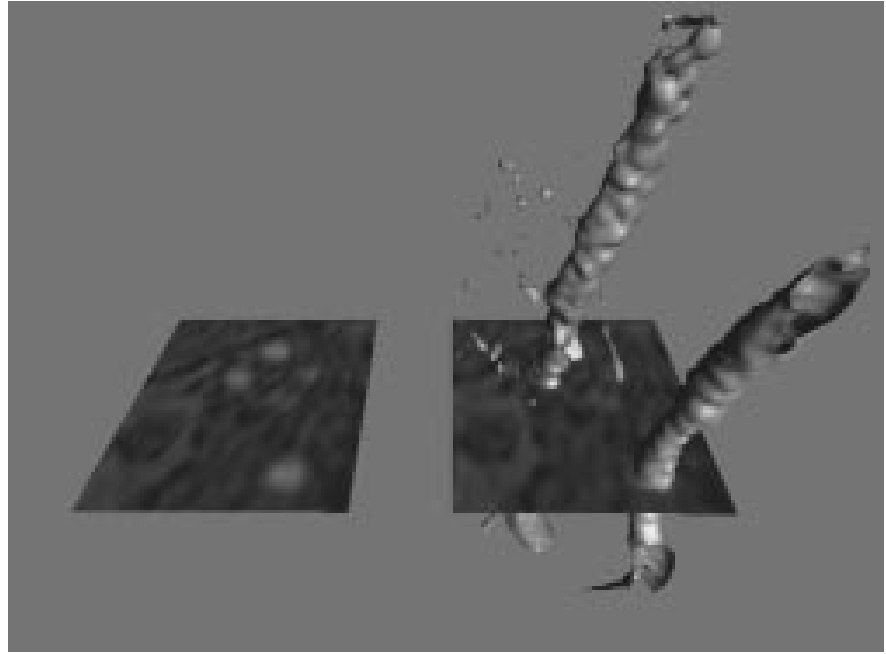

Fig. 7. Identifying points along the axis of the vessel. A cropped portion of the carotid MRA is shown by itself and with a 3-D isosurface (right) to provide anatomical context that helps to follow a given vessel from one axial slice to another.

\section{APPLICATION}

\section{A. Digital Phantom of Vessel With Stenosis}

A MRA image was simulated based on an idealistic geometry of a vessel with a stenosis. The geometry of the vessel is perfectly symmetrical about its axis. The radius of the vessel is described in Fig. 8. At the stenosis, the radius of the vessel tapers at a rate of $1 / 4$ pixels/pixel. The vessel tapers symmetrically for 12 pixel units in the axial direction to produce a percent stenosis of the vessel of $54.5 \%$ according to the clinical definition of stenosis

$$
\text { Percent Stenosis }=100\left(1-\frac{\text { minimum diameter }}{\text { normal diameter }}\right) \text {. }
$$

The 3-D image has isotropic voxel dimensions. A high-resolution binary mask of the vessel geometry is created. The binary mask is then down-sampled ten times in each direction. Each voxel in the resulting image is the sum of the binary values in the original image. This provides an image with the partial volume effects at the edges of the vessel. The image is blurred by convolution with 3-D Gaussian kernel with a space constant of 1.0 pixel units. After scaling, the maximal intensity within the vessel is 250 . Image noise is simulated using a pseudorandom number generator with a normal distribution. The image noise is blurred by convolution with a Gaussian with a space contant of 1.0 pixel units. After scaling, the standard deviation of the noise is 77. Cross-sectional views of the digital phantom are shown in Fig. 9.

The vessel surface of the digital phantom was reconstructed with the tubular deformable model using the axis of the idealistic shape and using a range of the smoothing parameter $K_{2}$. Values used for the smoothing parameter were $K_{4}=0.1,1$, 10,100 , and 1000 where $K_{4}=2.86 \times 10^{-4} K_{2}$. The iteration step size was $5 \times 10^{-3} \mathrm{~cm}$ and the 1000 deformation iterations were applied in the prevertex and postvertex merging phases (although no vertex merging occurs since the axis is straight in this case). The use of 1000 iterations is excessive but ensures the completion of the deformation process. The iteration step size 


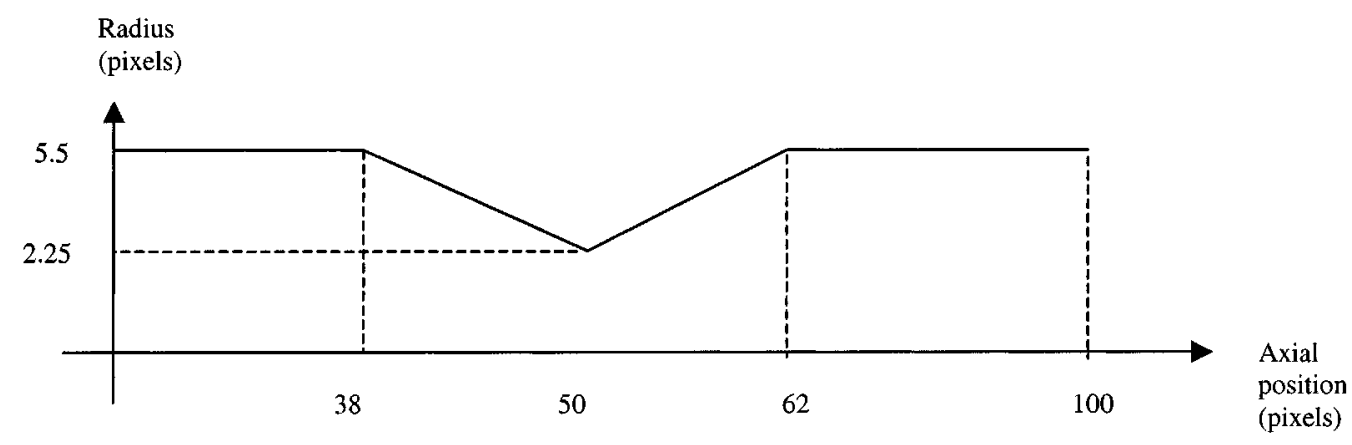

Fig. 8. Radius of idealistic vessel with stenosis. This vessel geometry is used for generating digital MRA phantom.

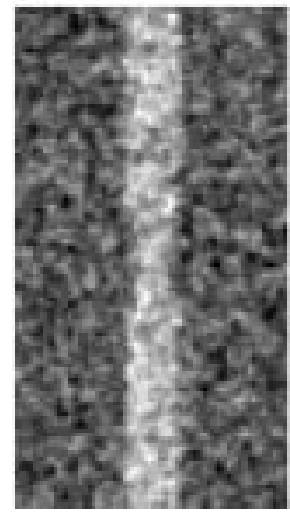

(a)

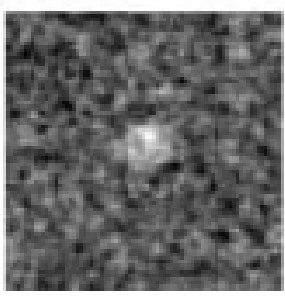

(b)

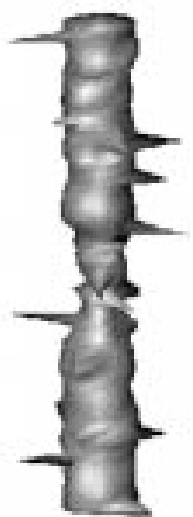

(c)

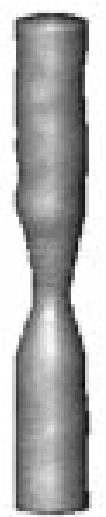

(d)

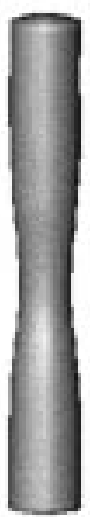

(d)

Fig. 9. Application of tubular deformable model to simulated MRA of vessel with stenosis. An artificial MRA image of a vessel with stenosis was created from an idealistic vessel shape. Image artifacts were incorporated into the image including normally distributed noise, the partial volume effect and blurring. (a) Longitudinal and (b) axial cross-sectional views of the image are shown. The results for surface reconstruction obtained using $K_{3}=0.1,100$, and 1000 are shown in (c), (d), and (e), respectively. Optimal surface reconstruction was obtained for $K_{3}=100$.

$K_{1}$ was chosen by trial-and-error. Obvious instabilities occur if the choice of $K_{1}$ is too large. The space constant of the gradient magnitude operator, $\nabla I(a, \varphi, r)$, used in the deformation [(3), (4), and (22), is $1.0 \mathrm{in}$-plane of in-plane pixel size. The results of the surface reconstruction are shown in Fig. 9(c)-(e)].

An average vessel radii was obtained at each axial position along the axis. To calculate the average radius, a cross-sectional area was calculated from the vertex positions at that radial position

$$
P_{j}=\sum_{i} T_{i}
$$

where $T_{i}$ is the triangular area between the axial point and two circumferentially adjacent points and $P_{j}$ is the polygonal area at the $j$ th axial location. The average radius is then given by the formula for the area of a circle multiplied by a constant factor, 1.025 , to compensate for the difference in area between a triangle and a corresponding arc of a circle

$$
\operatorname{radius}_{j}=\sqrt{\frac{1.025 P_{j}}{\pi}} .
$$

Twenty-five contiguous axial locations at both ends of the vessel were used to determine the average vessel radius and the standard deviation of the vessel radius. The measured degree of stenosis of the reconstructed vessel was defined as the minimum radius divided by the average vessel radius in the normal section of the vessel. The results are shown in Table I.
The surface was also reconstructed using vessel axes defined by user-interaction. For all trials $K_{3}$ was set to 100 . In all other respects, the surface reconstruction was identical to above study using idealistic vessel axis. The results of three trials are shown in Table II. The average radii of a segment was calculated from 8-10 axial points using (27). The average radii of all segments differed from the known radii by less than $20 \%$ of the average voxel dimension. Radii at the stenosis were compared with radii measured manually from computed tomography image (CT) of the phantom (15-cm field of view, reconstructed to $0.3-\mathrm{mm}$ in-plane resolution, Oriented normal to axis of stenosis). The difference between the radii measured by the deformable model and from CT was $0.2 \mathrm{~mm}$. The results of the surface reconstruction are shown in Fig. 10.

\section{B. Phantom of Carotid Artery Bifurcation With Stenosis}

The tubular deformable model was applied to an MR image of a glass phantom of the carotid artery bifurcation with stenosis of the internal CA(3-D SPGR, Voxel dimensions $0.31 \times 0.31 \times 1.0$ $\mathrm{mm}$, Phantom filled with 60:40 ratio water to glycerine, No fluid flow). The glass phantom was constructed according to typical dimensions $(\mathrm{CCA}=8 \mathrm{~mm}, \mathrm{ICA}=7 \mathrm{~mm}, \mathrm{ECA}=6 \mathrm{~mm})$ with a moderate stenosis at the origin of the ICA [27]. The CCA/ICA segment and CCA/ECA segments of the phantom were independently reconstructed from the MR image. For applications of the deformable model to images of the vessel phantom and 
TABLE I

EFFect of VARIATION OF SMOOTHING PARAMETER

\begin{tabular}{l|l|l|l}
\hline & $\begin{array}{l}\text { Normal Radius } \\
\text { (True radius is 5.5 } \\
\text { pixels) }\end{array}$ & $\begin{array}{l}\text { Standard Deviation of } \\
\text { normal radius }\end{array}$ & $\begin{array}{l}\text { Degree of stenosis } \\
\text { (True stenosis is } \\
54.5 \%)\end{array}$ \\
\hline $\begin{array}{l}K_{4}=0.1 \\
\left(K_{2}=3.5 \times 10^{2} \mathrm{~cm}^{-1}\right)\end{array}$ & 5.37 pixels & 0.273 pixels & $51.4 \%$ \\
\hline $\begin{array}{l}K_{4}=1 \\
\left(K_{2}=3.5 \times 10^{3} \mathrm{~cm}^{-1}\right)\end{array}$ & 5.31 & 0.193 & 51.2 \\
\hline $\begin{array}{l}K_{4}=10 \\
\left(K_{2}=3.5 \times 10^{4} \mathrm{~cm}^{-1}\right)\end{array}$ & 5.29 & 0.128 & 54.7 \\
\hline $\begin{array}{l}K_{4}=100 \\
\left(K_{2}=3.5 \times 10^{5} \mathrm{~cm}^{-1}\right)\end{array}$ & 5.28 & 0.106 & 48.8 \\
\hline $\begin{array}{l}K_{4}=1000 \\
\left(K_{2}=3.5 \times 10^{6} \mathrm{~cm}^{-1}\right)\end{array}$ & 5.23 & 0.090 & 28.4 \\
\hline
\end{tabular}

TABLE II

Variability of Surface Reconstruction Due to Manual Definition of Vessel Axis

\begin{tabular}{l|l|l|l}
\hline & $\begin{array}{l}\text { Normal Radius } \\
\text { (True radius is 5.5 } \\
\text { pixels) }\end{array}$ & $\begin{array}{l}\text { Standard Deviation of } \\
\text { normal radius } \\
\text { (\% of true radius) }\end{array}$ & $\begin{array}{l}\text { Degree of stenosis } \\
\text { (True stenosis is } \\
54.5 \%)\end{array}$ \\
\hline Trial 1 & 5.23 pixels & 0.122 pixels & $48.3 \%$ \\
\hline Trial 2 & 5.22 & 0.130 & 47.9 \\
\hline Trial 3 & 5.26 & 0.0831 & 48.4 \\
\hline
\end{tabular}

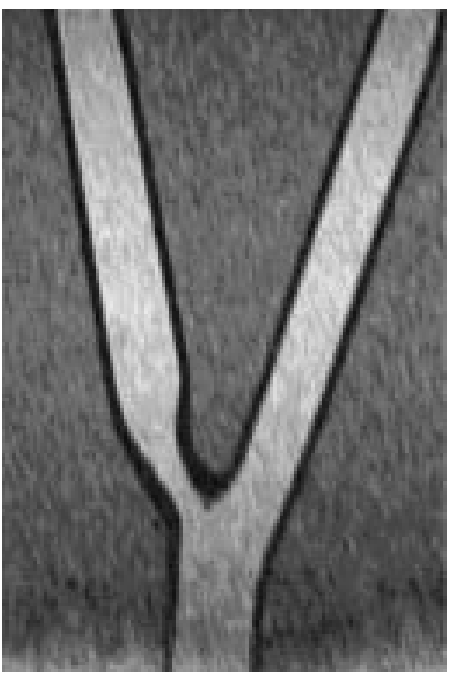

(a)

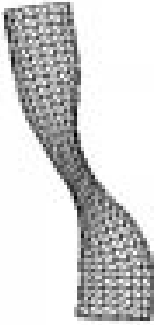

(b)

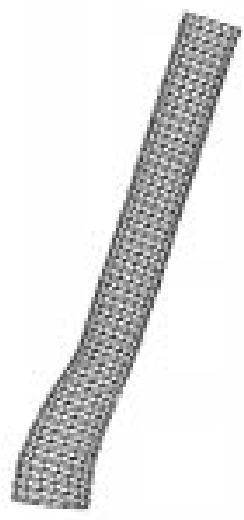

(c)

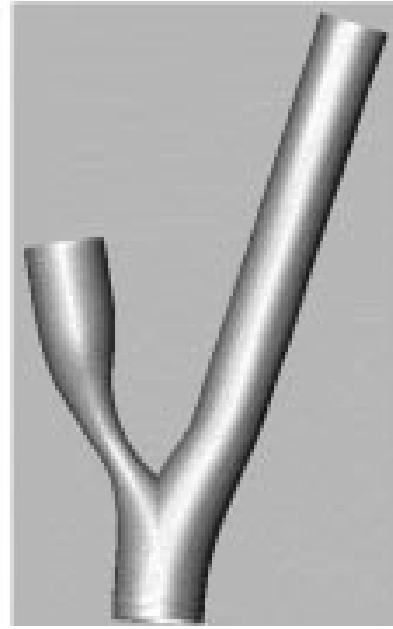

(d)

Fig. 10. Surface reconstruction of phantom of carotid bifurcation. The carotid artery bifurcation is reconstructed from an MR image of a glass phantom. (a) Single slice of image. (b) and (c) The surface was reconstructed with the tubular deformable model of overlapping segments. (d) The superimposition of the two surfaces accurately represents the bifurcation region.

to MRA, the elasticitiy parameter $K_{2}$ was chosen by the process of trial-and-error although the same value, $K_{2}=7 \times 10^{3} \mathrm{~cm}^{-1}$ is used for both the phantom and data from human subjects The space constant of the gradient magnitude operator, $\nabla I(a, \varphi, r)$, is 1.0 in-plane voxel units for all tests of the algorithm. Image contrast is similar for all images. The contrast is in the range of 200 to 300 gray-scale units. The reconstruction of the phantom is carried out in a total of 100 deformation iterations. Fifty iterations are carried out prior to merging of vertices and 50 after merging of vertices. Visible change in the surface was not seen beyond this number of iterations. The choice of the iteration step size $K_{1}$ was made so as to avoid a computational instability that is analogous to an undamped oscillation of the mechanical spring system. The iteration step size application to all MR images was $K_{1}=0.05 \mathrm{~cm}$.

\section{Carotid Arteries of Normal Subjects}

Surface reconstruction of the carotid artery was carried out on contrast-enhanced MRA images. MR images were acquired with contrast injection of Gd-DTPA (fast-gradient recalled echo sequence, GE Corp.) of one carotid artery from each of four normal subjects. As with reconstruction of phantom, the reconstruction was carried out with 50 deformations prior to merging 


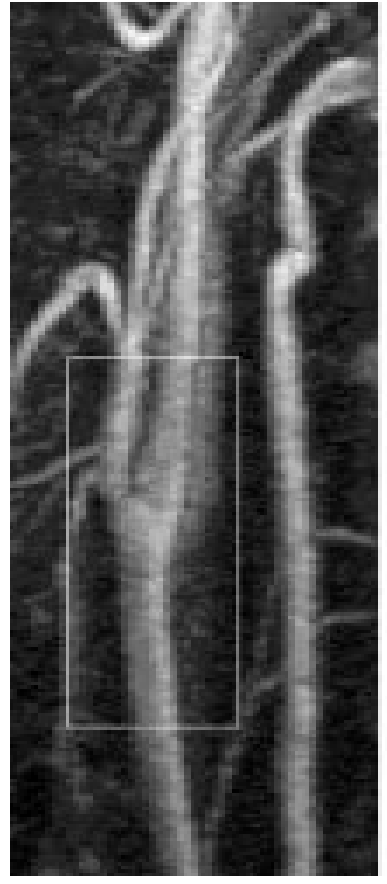

(a)

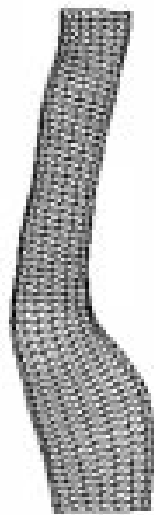

(b)

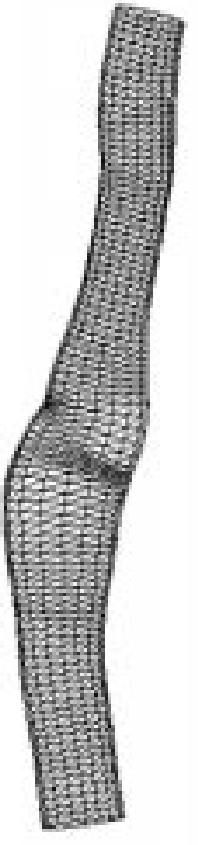

(c)

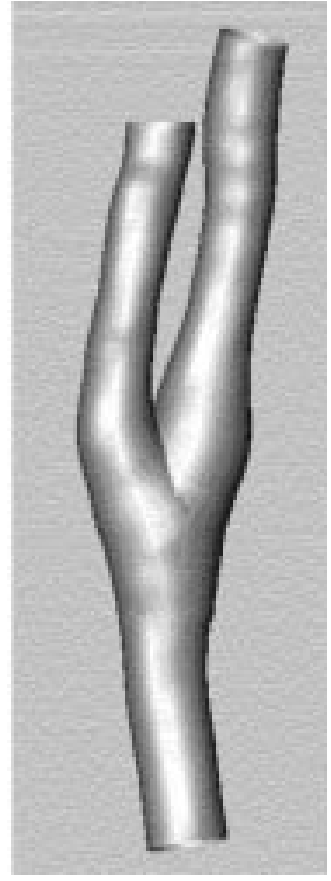

(d)

Fig. 11. Surface reconstruction of carotid artery bifurcation from normal subject. (a) Cropped portion of the MRA is visualized as a MIP in the sagital view. (b) CCA/ECA segment. (c) CCA/ICA segment are reconstructed separately and (d) superimposed as a shaded surface display.

of vertices and 50 after merging. The iteration step size $K_{1}=$ $0.05 \mathrm{~cm}$, was also the same as for the phantom reconstruction.

Realistic surfaces were obtained for all four images that were consistent with both the MIP's and the source images (Fig. 11). The vessels all exhibited the characteristic bulb at the origin of the internal carotid artery. Also, the relative diameters of the common, internal and external carotid arteries are consistent with the normal anatomy. Only a limited portion of ECA and its sub-tree was reconstructed since the vessel rapidly bifurcates which increases the complexity of identifying the axis. However, the portion of the ECA reconstructed is adequate for CFD modeling of flow in the carotid bifurcation.

\section{Carotid Arteries With Stenosis}

Two carotid arteries from each of two subjects with stenosis of the carotid artery were reconstructed with the tubular deformable model. The result for one of the vessels is shown in Fig. 12. For one image, the reconstructed surface is smooth and entirely consistent with the MIP and source images. For the other image (the one shown in Fig. 12), the surface reconstruction has one noticeable error at the origin of the ICA. At this location, an indentation in the lumen was smoothed-over in the reconstruction. This error occurred due to small size of the indentation and due to proximity to the insertion of the ECA where there is a discontinuity in the radial location of the surface. This error can be manually corrected by inserting a point to provide an attractive force toward the correct location. The elastic coefficient of the manual attractive force $K_{3}=7 \times 10^{4} \mathrm{~cm}^{-1}$ [in (4)] as determined by trial-and-error. When a manual attractive force is present the iteration step size was reduced to, $K_{1}=$ $0.025 \mathrm{~cm}$ to avoid instability in the simulation of the mechanical deformation. The total number of deformation iterations is then increased to a total of 100 .
The degree of stenosis apparent in the surface reconstruction is less than in the MIP. However, the discrepancy is not due to purely to smoothing effects of the deformable model since a similar degree of stenosis is observed when the surface is constructed with no smoothing. The error may be attributed to the tendency of the MIP to over-estimate the degree of stenosis. Only one artery from each subject was reconstructed since the contra-lateral vessel was occluded in one case and suffered from severe image artifacts in the other.

\section{E. Renal Arteries With Stenosis}

The abdominal aorta and renal artery were reconstructed in two subjects with renal artery stenosis from contrast-enhanced MRA (fast gradient recalled echo sequence, GE Corp.). The surface reconstruction parameters were the same as those used for the carotid arteries. Surfaces of both the renal arteries and the aortas are smooth and are consistent with MIP and source images. The results are shown in Fig. 13.

\section{DISCUSSION}

The tubular deformable model is an attractive method for blood-vessel surface reconstruction from 3-D angiography. This method is similar to the Frangi model [26] for vessel surface reconstruction. Like the Frangi model, for example, the surface mesh of our model is cylindrical. However, the two deformable models differ in important respects. One important difference, which favors our method, is the process of initialization of the deformable model. The multiscale method used by Frangi et al. (and proposed earlier by Aylward et al. [28]) requires a consistent cross-sectional image intensity profile along blood vessels. However, for contrast-enhanced MRA, flow and timing artifacts 


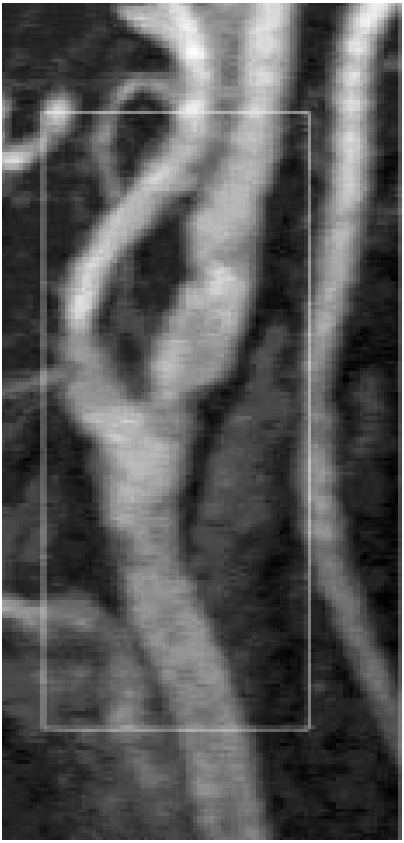

(a)

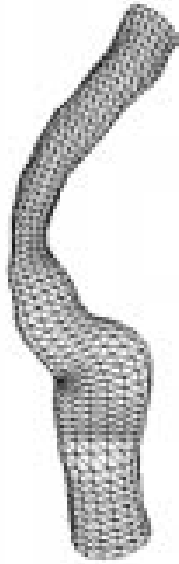

(b)

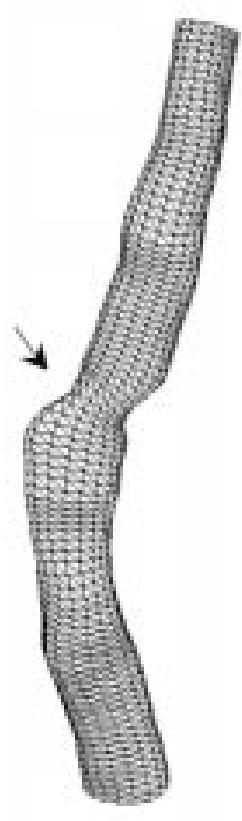

(c)

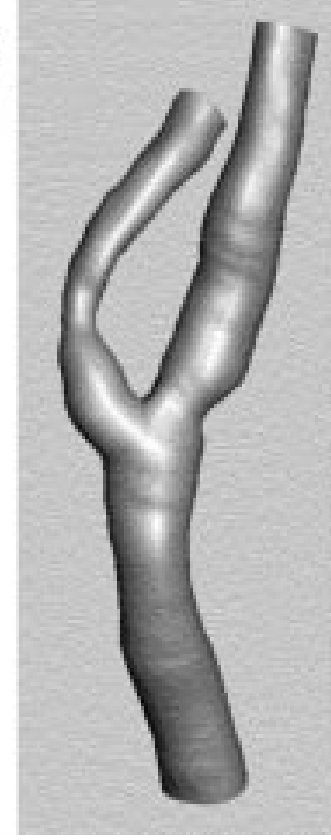

(d)

Fig. 12. Surface reconstruction of carotid artery bifurcation. (a) Carotid artery bifurcation from contrast-enhanced MRA is reconstructed as one segment continued onto (b) the ECA and one onto (c) the ICA. (d) The two surfaces are superimposed. The surface of the internal segment was manually indented at the groove between the internal and external carotid arteries [arrow in (c)] where an error otherwise occurs due to the discontinuity of the tubular shape at that point.

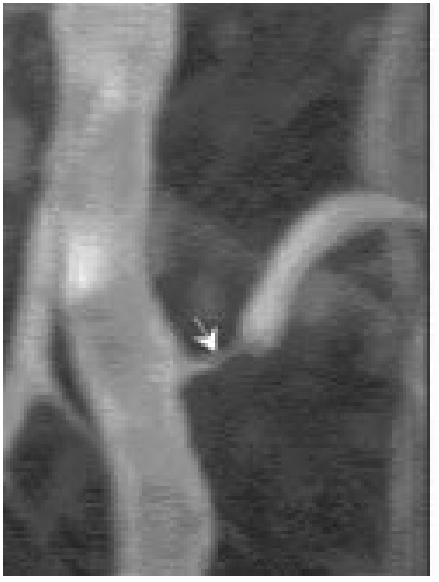

(a)

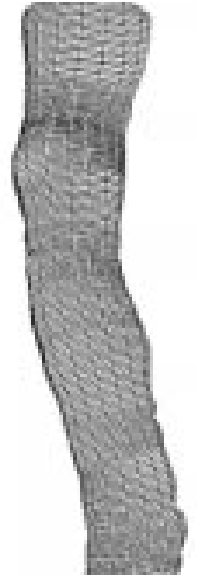

(b)

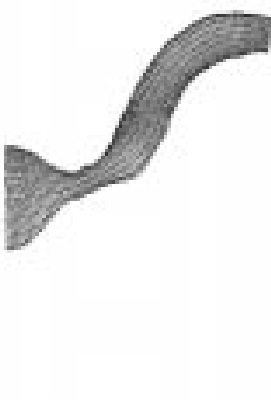

(c)

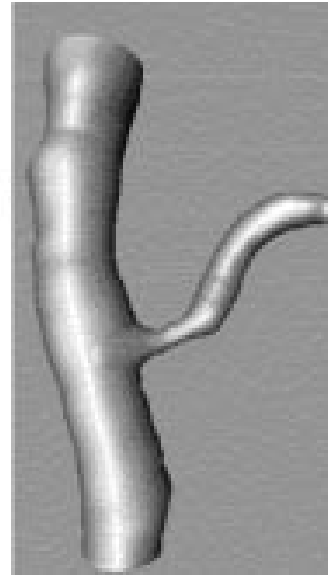

(d)

Fig. 13. Surface reconstruction of a renal artery with stenosis. (a) Tubular deformable model applied to MRA of patient with renal artery stenosis. Reconstruction performed independently on (b) aorta and (c) left renal artery. (d) Vessels superimposed and visualized as shaded surface display.

can significantly distort the cross-sectional image intensity profile. For example, the jugular vein, which is immediately adjacent to the carotid artery will enhance to varying degrees depending on the rates of venous return in the given individual and to the exact timing of the image acquisition. The presence of the vein next to carotid artery will affect the localization of the axis of an object using multiscale methods [29]. Inititialization of the tubular deformable model is a 1D edge detection method which assumes only the image intensity decrease is greatest at the boundary of the lumen.

Another advantage of our method is that meshes are constructed with high vertex density. This allows for representation of smaller-scale detail of the lumen shape such as focal stensoses or protrusions into or out from the lumen. High vertex density cannot be obtained for simple cylindrical meshes where vertices are simply placed along normals from the axis. For such a cylindrical mesh, the radial lines from nearby points along the axis will converge at the inside of curves of the cylindrical axis and intersect with one another. This problem is avoided in the tubular coordinate system by a systematic merging of radial lines.

The tubular coordinate system allows for the description of the vessel shape in terms of a single radial parametric function. This has a subtle advantage over the rectilinear parametric functions used in the Frangi model [26]. Whereas smoothing of rectilinear parametric functions of shape must be biased toward either under- or over-estimation of vessel diameter, no such bias is present in the tubular deformable model. While the rectilinear parametric shape functions allow for reconstruction of more generalized shapes, that is only a modest advantage for the reconstruction of blood vessel surfaces. 
Vessel bifurcations can be accurately reconstructed with the tubular deformable model. While we have only shown the bifurcations as superimposed 3-D shaded surface displays, the surface meshes themselves can be merged. A straightforward method for merging such surfaces has recently been developed [30].

\section{CONCLUSION}

The tubular deformable model will be useful for quantification of vessel shape from 3-D angiograph. Particularly in cases where there is a mild rate of tapering of the lumen at a stenosis, the tubular deformable model is likely to provide a more accurate measurement of the degree of stenosis than from manual interpretation. The deformable model will also enable more accurate realistic CFD modeling of blood flow patterns and better assessment of the risk of stroke, for example, posed by a given abnormality in the carotid lumen shape.

Another potential application of the tubular deformable model is for artery-vein separation in contrast-enhanced MRA. The tubular deformable model could conceivably be applied to a vein nearby to an artery of interest. Once the surface of the vein is reconstructed, it could be nulled-out to leave an unobstructed view of the artery. These potential applications will be addressed in future investigations.

The method we propose for surface reconstruction requires that the vessel axis be defined manually. This is a satisfactory approach since the central point of a vessel can be manually identified relatively quickly and accurately. However, a greater degree of automation in the vessel axis definition is desirable. We do not address this problem in this paper since the axis detection step is largely independent from the surface reconstruction process. However, there has recently been significant progress in this area [31], [28], [32].

\section{REFERENCES}

[1] North American Symptomatic Carotid Trial (NASCET) Steering Committee, "North american symptomatic carotid endarterectomy trial; methods, patient characteristics and progress," Stroke, vol. 22, pp. 711-720, 1991.

[2] T. Scarabina, A. Carriero, G. M. Giannatempo, and R. Marano et al., "Contrast-enhanced MR angiography (CE MRA) in the study of the carotid stenosis: Comparison with digital subtraction angiography (DSA)," J. Neuroradiol., vol. 26, pp. 87-91, 1999.

[3] C. P. Allott, C. D. Barry, R. Pickford, and J. C. Waterton, "Volumetric assessment of carotid artery bifurcation using freehand-acquired, compound 3-D ultrasound," Br. J. Radiol., vol. 72, pp. 289-292, 1999.

[4] M. E. Cinat, H. Pham, D. Vo, I. Gordon, and S. E. Wilson, "Improved imaging of carotid artery bifurcation using helical computed tomographic angiography," Ann. Vasc. Surg., vol. 13, pp. 178-183, 1999.

[5] J. A. Moore, D. A. Steinman, D. W. Holdsworth, and C. R. Ethier, "Accuracy of computational hemodynamics in complex arterial geometries reconstructed from magnetic resonance imaging," Ann. Biomed. Eng., vol. 27, pp. 32-41, 1999.

[6] Q. Long, X. Y. Xu, M. Bourne, and T. M. Griffith, "Numerical study of blood flow in an anatomically realistic aorto-iliac bifurcation generated from MRI data," Magn. Reson. Med., vol. 43, pp. 565-576, 2000.

[7] K. C. Wang, R. W. Dutton, and C. A. Taylor, "Improving geometric model contruction for blood flow modeling," IEEE Eng. Med. Biol., Mag., vol. 18, pp. 33-39, 1999.

[8] R. White, Ed., Atherosclerosis and Arteriosclerosis: Human Pathology and Experimental Animal Methods and Models. Boca Raton, FL: CRC, 1989.

[9] B. E. Sumpio, Hemodynamic Forces and Vascular Cell Biology. Austin, TX: Landes, 1993.

[10] H. E. Cline, W. E. Lorensen, S. P. Souza, F. A. Jolesz, R. Kikinis, G. Gerig, and T. E. Kennedy, "3-D surface rendered MR images of the brain and its vasculature," JCAT, vol. 15, pp. 344-351, 1991.
[11] T. K. Foo, V. B. Ho, and P. L. Choyke, "Contrast-enhanced carotid MR angiography. Imaging principles and physics," Neuroimag. Clin. $N$ Amer, vol. 9, pp. 263-284, 1999.

[12] D. L. Wilson and J. A. Noble, "Segmentation of cerebral vessels and aneurysms from MR angiography data," in Lecture Notes in Computer Science. Berlin, Germany: Springer-Verlag, 1997, vol. 1230, pp. 423-428.

[13] T. Lei, J. K. Udupa, P. K. Saha, and D. Odhner, "MR angiographic visualization and artery-vein separation," Proc. SPIE, vol. 3658, pp. 58-66, 1999.

[14] P. J. Yim, D. Kim, and C. Lucas, "High resolution four-dimensional surface reconstruction of the right heart and pulmonary arteries," Proc. SPIE, vol. 3338, pp. 726-738, 1998.

[15] P. J. Yim and R. M. Summers, "Analytic surface reconstruction by local threshold estimation in the case of simple intensity contrasts," Proc. SPIE, vol. 3660, pp. 288-300, 1999.

[16] J. R. Cebral and R. Lohner, "From medical images to CFD meshes," in Proc. 8th Int. Meshing Roundtable, So. Lake Tahoe, CA, 1999, pp. 321-332.

[17] T. Lei, J. K. Udupa, P. K. Saha, and D. Odhner, "MR angiographic visualization and artery-vein separation," Proc. SPIE, vol. 3658, pp. 58-66, 1999.

[18] S. Aylward, E. Bullit, S. Pizer, and D. Eberly, "Intensity ridge and widths for tubular object segmentation and description," presented at the IEEE Workshop, Mathematical Methods in Biomedical Image Analysis, June 1996.

[19] Y. Sato, S. Nakajima, H. Atsumi, T. Koller, G. Gerig, S. Yoshida, and R. Kikinis, "3-D multi-scale line filter for segmentation and visualization of curvilinear structures in medical images," in Lecture Notes in Computer Science. Berlin, Germany: Springer-Verlag, 1997, vol. 1205, pp. 213-222.

[20] C. Lorenz, I.-C. Carlsen, T. M. Buzug, C. Fassnacht, and J. Weese, "Multi-scale line segmentation with automatic estimation of width, contrast, and tangential direction in 2-D and 3-D medical images," in Lecture Notes in Computer Science. Berlin, Germany: Springer-Verlag, 1997, vol. 1205, pp. 233-242.

[21] T. McInerney and D. Terzopoulos, "Deformable models in medical image analysis: A survey," Med. Image Anal., vol. 1, pp. 91-108, 1996.

[22] Y. L. Hu, W. J. Rogers, D. A. Coast, C. M. Kramer, and N. Reichek, "Vessel boundary extraction based on a global and local deformable physical model with variable stiffness," Magn. Reson. Imag., vol. 16, pp. 943-951, 1998.

[23] J. D. Gill, H. M. Ladak, D. A. Steinman, and A. Fenster, "Accuracy and variability assessment of a semiautomatic technique for segmentation of the carotid arteries from three-dimensional ultrasound images," Med. Phys., vol. 27, pp. 1333-1342, 2000

[24] T. McInerney and D. Terzopoulos, "Medical image segmentation using topologically adaptable surfaces," presented at the CVRMed'97, Grenoble, France, Mar. 1997.

[25] A. J. Bulpitt and E. Berry, "Spiral CT of abdominal aneurysms: Comparison of segmentation with an automatic 3-D deformable model and interactive segmentation," Proc. SPIE, vol. 3338, pp. 938-946, 1998.

[26] A. F. Frangi, W. J. Niessen, and R. M. Hoogeveen, "Model-based quantitation of 3-D magnetic resonance angiographic images," IEEE Trans. Med. Imag., vol. 18, pp. 946-956, Oct. 1999.

[27] R. F. Smith, B. K. Rutt, A. J. Fox, and R. N. Rankin, "Geometric characterization of stenosed human carotid arteries," Acad. Radiol., vol. 3 , pp. 989-911, 1996.

[28] S. Aylward, E. Bullit, S. Pizer, and D. Eberly, "Intensity ridge and widths for tubular object segmentation and description," presented at the IEEE Workshop, Mathematical Methods in Biomedical Image Analysis, June 1996.

[29] M. McAuliffe, D. Eberly, D. S. Fritsch, E. L. Chaney, and S. M. Pizer, "Scale-space boundary evolution initialized by cores," in Lecture Notes in Computer Science, K. H. Hvhne and R. Kikinis, Eds. Berlin, Germany: Springer-Verlag, 1996, vol. 1131, Visualization in Biomedical Computing, pp. 173-182.

[30] J. Cebral, R. Lohner, P. L. Choyke, and P. J. Yim, "Merging intersecting triangulations for finite element modeling," J. Biomech., vol. 34, pp. 815-819, 2001.

[31] O. Wink, W. J. Niessen, and M. A. Viergever, "Fast delineation and visualization of vessels in 3-D angiographic images," IEEE Trans. Med. Imag., vol. 19, p. 337, Apr. 2000.

[32] P. J. Yim, R. M. Summers, and P. L. Choyke, "Gray-scale skeletonization of small vessels in magnetic resonance angiograms," IEEE Trans. Med. Imag., vol. 19, pp. 568-576, June 2000. 